\title{
Hepatite C em portadores do vírus HIV-1 usuários de drogas no Piauí, Brasil
}

\author{
Hepatitis C in HIV-1 virus users using drugs in Piauí, Brazil \\ Hepatitis C en usuarios del virus VIH-1 que usan drogas en Piauí, Brasil
}

Recebido: 29/12/2020 | Revisado: 03/01/2021 | Aceito: 05/01/2021 | Publicado: 06/01/2021

\author{
Sâmia Moreira de Andrade \\ ORCID: https://orcid.org/0000-0002-2310-2515 \\ Centro Universitário Santo Agostinho, Brasil \\ E-mail: samia.andrade27@hotmail.com \\ Evaldo Hipólito de Oliveira \\ ORCID: http://orcid.org/0000-0003-4180-012X \\ Universidade Federal do Piaú, Brasil \\ E-mail: evaldohipolito@gmail.com
}

\begin{abstract}
Resumo
Esta pesquisa científica objetivou avaliar e identificar importantes aspectos epidemiológicos das infecções pelo HCV e HIV em pacientes HIV-Aids usuários de drogas ilícitas no estado do Piauí, como também, identificar fatores de risco à coinfecção. A pesquisa foi desenvolvida em duas etapas: (1) Formação de um banco de dados a partir de dados secundários e (2) Análise/Divulgação. A $1^{\mathrm{a}}$ etapa do estudo foi constituída pela formação de um banco de dados a partir de informações epidemiológicas e diagnóstica da população de pacientes infectadas pelo vírus HIV usuária de drogas no estado do Piauí. Já a $2^{\mathrm{a}}$ etapa do estudo foi constituída pela análise dos dados coletados na $1^{\mathrm{a}}$ fase e início da divulgação dos resultados do estudo. As variáveis sociodemográficas dos indivíduos portadores do HIV/HCV no Piauí apresentaram-se mais alta no sexo masculino, idade acima de 40 anos, cor preta/parda. Enquanto à associação significativa referente à exposição à infecção pelo vírus HIV no Piauí, observa uma maior relevância para relacionamento sexual com usuário de ilícita, cirurgia, preferência sexual e DST. Sendo que ambos contribuem para a evolução da epidemia, principalmente por se relacionar a comportamento sexual de risco. Não sendo possível identificar um único fator responsável pela coinfecção. Em suma, esta proposta contribuiu com informações epidemiológicas relevantes sobre a população de pacientes HIV-Aids usuária de drogas ilícitas e as infecções ocasionadas pelos vírus HIV/HCV.
\end{abstract}

Palavras-chave: Hepatite C; Usuários de drogas; HIV; Coinfecção.

\begin{abstract}
This scientific research aimed to assess and identify important epidemiological aspects of HCV and HIV infections in HIV-AIDS patients using illicit drugs in the state of Piauí, as well as to identify risk factors for co-infection. The research was developed in two stages: (1) Formation of a database from secondary data and (2) Analysis / Disclosure. The first stage of the study consisted of the formation of a database based on epidemiological information and diagnosis of the population of patients infected with the HIV virus using drugs in the state of Piauí. The 2nd stage of the study, on the other hand, consisted of analyzing the data collected in the 1st stage and beginning to disclose the results of the study. The socio-demographic variables of individuals with HIV / HCV in Piauí were higher in males, aged over 40 years, black/brown. As for the significant association regarding exposure to HIV infection in Piauí, there is a greater relevance for sexual relationships with users of illicit drugs, surgery, sexual preference and STDs. Both of which contribute to the evolution of the epidemic, mainly because it is related to risky sexual behavior. It is not possible to identify a single factor responsible for co-infection. In short, this proposal contributed with relevant epidemiological information on the population of HIV-AIDS patients using illicit drugs and infections caused by the HIV / HCV viruses.
\end{abstract}

Keywords: Hepatitis C; Drug users; HIV; Coinfection.

\section{Resumen}

Esta investigación científica tuvo como objetivo evaluar e identificar aspectos epidemiológicos importantes de las infecciones por VHC y VIH en pacientes con VIH-SIDA que utilizan drogas ilícitas en el estado de Piauí, así como identificar factores de riesgo de coinfección. La investigación se desarrolló en dos etapas: (1) Formación de una base de datos a partir de datos secundarios y (2) Análisis / Divulgación. La primera etapa del estudio consistió en la conformación de una base de datos basada en información epidemiológica y diagnóstico de la población de pacientes infectados por el virus del VIH que usan drogas en el estado de Piauí. La $2^{\mathrm{a}}$ etapa del estudio, en cambio, consistió en analizar los datos recolectados en la $1^{\mathrm{a}}$ etapa y comenzar a divulgar los resultados del estudio. Las variables sociodemográficas de las personas con VIH/VHC en Piauí fueron mayores en los hombres, mayores de 40 años, negro / moreno. En cuanto a la asociación significativa con respecto a la exposición a la infección por VIH en Piauí, existe una mayor relevancia para las relaciones sexuales con usuarios de drogas ilícitas, cirugía, preferencia sexual y ETS. Ambos contribuyen a la evolución de la epidemia, principalmente porque está relacionada con conductas sexuales de 
riesgo. No es posible identificar un solo factor responsable de la coinfección. En definitiva, esta propuesta aportó información epidemiológica relevante sobre la población de pacientes VIH-SIDA que consumen drogas ilícitas y las infecciones causadas por los virus VIH / VHC.

Palabras clave: Hepatitis C; Drogadictos; VIH; Coinfección.

\section{Introdução}

A infecção pelo vírus da hepatite $\mathrm{C}(\mathrm{HCV})$ em portadores do vírus da imunodeficiência humana (HIV) é um importante problema de saúde pública no Brasil e no mundo. Atualmente, o ato sexual e o compartilhamento de equipamentos para o uso de drogas ilícitas (UDI) são os principais meios de transmissão destes vírus (Kuo, 2012).

Pode ser observada a infecção de pacientes por mais de um vírus, frequentemente a coinfecção do vírus da Hepatite C (HCV) em portadores do vírus HIV, em virtude de estes compartilharem os mesmos mecanismos de transmissão (parenteral, sexual e vertical). Essa semelhança epidemiológica explica a alta frequência da coinfecção pelos dois vírus (Calegari et al. 2011). A presença do HCV no portador do vírus HIV reveste-se de importância clínica, na medida em que a ocorrência de tal coinfecção parece favorecer um pior prognóstico do paciente, bem como interferir nos resultados da terapêutica aplicada (Abreu et al., 2013).

Entre os grupos de risco, os usuários de drogas ilícitas (UDI) se destacam pela exposição a muitas situações que possibilitam a transmissão do vírus. Pela via sanguínea, a transmissão do HCV entre UDI pode ocorrer por meio de compartilhamento de objetos contaminados (agulhas, seringas, lâmina de barbear e alicate de unha) e demais equipamentos para o uso de drogas endovenosas e inalatórias. Há também o risco de transmissão através de acidentes com perfurocortantes, procedimentos cirúrgicos, odontológicos e os politransfundidos (Nelson et al., 2011).

A prostituição, a promiscuidade sexual, a elevada frequência de relação sexual anal não protegida aumentam a chance de aquisição do vírus, com riscos aumentados nas relações sexuais anais, reduzindo gradativamente até o sexo oral. Portanto, a relação sexual anal, tanto insertiva como receptiva, eleva os índices de infecção pelo HCV e HIV (Carvalho et al., 2009). E principalmente, a ausência de preservativo durante a relação sexual constitui os principais comportamentos de risco às infecções pelo HCV e HIV e as práticas e situações a ela relacionadas, aí incluídos o consumo de drogas ilícitas e de bebidas alcoólicas, a exposição a prisões, o baixo nível educacional e marginalização socioeconômica comumente detectadas em população de usuários de drogas ilícitas em diversas partes do mundo (Santos et al., 2008).

O uso inconsciente de preservativos entre os UDI e seus parceiros, que em muitos dos casos, não eram usuários de drogas injetáveis fez com que a epidemia se disseminasse a partir dessa população específica para a população dita geral. Desta forma, parceiros de UDI representam uma "ponte" para a disseminação do HIV e HCV entre esses segmentos (Reis, 2010).

Diversos estudos realizados apontam maiores prevalências em grupos populacionais expostos a fatores de risco específicos, como indivíduos infectados pelo HIV, tanto homossexuais como heterossexuais elevam o risco à coinfecção pelo HCV (Carvalho et al., 2009).

A interação destes vírus é preocupante e constitui um dos problemas mais importantes de saúde pública a ser enfrentado por profissionais de saúde e autoridades em todo o mundo. Atualmente, a caracterização epidemiológica da população de usuários de drogas ilícitas infectados pelo vírus HIV é uma ferramenta importante para combater e controlar a infecção e dependência química (Aquino et al., 2008).

Outro aspecto importante, é que, mesmo nos países onde a doença é de notificação compulsória, estima-se um grande volume de subnotificações de casos, explicando, em parte, pela associação entre infecção e grupos socialmente marginalizados, como usuários de drogas e homossexuais. Além disso, as hepatites virais, por produzirem apreciável contingente de infecções assintomáticas, acabam por se disseminar na coletividade de uma maneira mais intensa que a evidenciada pelo aparecimento de casos clínicos, e por isso deixam de ser diagnosticadas (Silva \& Barone, 2006). 
Vale ressaltar que o uso de drogas não-injetáveis tais como a cocaína inalada e o crack se relacionam a um comportamento sexual de risco, a prática do uso de drogas inalada com compartilhamento de canudo também pode veicular sangue pela escarificação de mucosa. Enquanto a transmissão viral entre usuários de drogas injetáveis é primariamente parenteral, através do compartilhamento dos equipamentos de injeção. Qualquer procedimento que envolva sangue pode servir de mecanismo de transmissão desses vírus, quando os instrumentos utilizados não forem devidamente limpos e esterilizados (Strazza et al., 2007).

Usuários de drogas representam uma população bastante vulnerável à infecção pelo HIV, seja pelas próprias formas de consumo destas substâncias - via parenteral, com compartilhamento de equipamentos de injeção (e, em menor escala, pelo compartilhamento de apetrechos e matérias no uso não parenteral, como canudos, cachimbos para fumar o crack etc.), seja pela pratica do sexo não-seguro, ou pela superposição desses dois fatores: por exemplo, devido ao fato de algumas drogas agirem como estimulante sexual ou, em algumas situações, pelo fato de a atividade sexual ser um meio para se conseguir um objetivo: a aquisição de drogas (Reis, 2010).

Desse modo, esta proposta de pesquisa revelará importantes aspectos epidemiológicos da infecção pelo HCV em UD no Estado do Piauí e determinará características demográficas, sociais, econômicas e de uso de drogas da população alvo do estudo, o que possibilitará o aperfeiçoamento das estratégias e políticas públicas no estado de controle e prevenção ao uso de drogas ilícitas. Desta forma, esta pesquisa científica objetivou avaliar e identificar importantes aspectos epidemiológicos das infecções pelo HCV e HIV em pacientes HIV-Aids usuários de drogas ilícitas no estado do Piauí, como também, identificar fatores de risco à coinfecção.

\section{Metodologia}

Foi realizada uma pesquisa transversal, epidemiológica, quantitativa, retrospectiva documental desenvolvido em duas etapas (Pereira et al., 2018).

Etapa 1: Formação de um banco de dados a partir de dados secundários. Essa etapa teve duração de dois meses e foi constituída pelas informações epidemiológicas, sociodemográficas, diagnóstico sorológico e molecular das infecções pelos vírus HCV em pacientes HIV-Aids usuários de drogas-UD. Foram utilizados artigos sobre o tema, encontrados nas bases de dados Scielo e Bireme, dissertações de mestrado e revistas.

Etapa 2: Análise e Divulgação. Essa etapa teve duração de três meses e foi constituída pela análise das informações obtidas na etapa 1 do estudo. Além disso, cada variável epidemiológica foi analisada isoladamente com o seu respectivo diagnóstico para infecção pelo HCV por regressão logística simples.

\section{População e Amostra, Critérios de Inclusão e Exclusão}

A nossa população foi constituída de 2.600 indivíduos portadores do vírus HIV, que após adotar os critérios de inclusão e exclusão, foram utilizados 214 indivíduos portadores do vírus HIV, usuários de drogas a partir do banco de dados. Os critérios de inclusão foram:

- Portadores do vírus HIV-Aids, que estão em monitoramento de carga viral e contagem de células CD4 e CD8.

- Usuários de drogas injetáveis e não injetáveis.

Tendo como critérios de exclusão:

- Portadores do vírus HIV-Aids com idade inferior de 18 anos. 
-Portadores do vírus HIV-Aids pertencentes a outros estados que não o Estado do Piauí.

\section{Coletas de Dados}

A formação de bancos de dados foi feito a partir dos arquivados depositados no Laboratório de Microbiologia Clínica, do Curso de Farmácia da Universidade Federal do Piauí-UFPI, construído do período de janeiro a maio de 2010.

\section{Análises de Dados}

A análise por regressão logística múltipla foi feita para determinar a associação independente e controlada de cada possível fator de risco com o diagnóstico viral. O nível de significância do risco foi avaliado pelo valor de $(P<0,05)$, e no Teste Exato de Fisher e a precisão, pelo intervalo de confiança de $95 \%$, calculados para cada categoria das variáveis presentes no estudo e cálculos de Odds Ratio (OR). A análise bivariada foi realizada através do teste do Quiquadrado $\left(\chi^{2}\right)$. Para a aceitação das hipóteses alternativas, foi considerado um intervalo de confiança de $95 \%$, com nível de significância estatística de $\mathrm{p}<0,05$. A tabulação e a análise estatística foram realizadas pelo programa SPSS for Windows, versão 20.0 (SPSS Inc, Chicago, Estados Unidos).

\section{Critérios Éticos}

Este projeto de pesquisa foi submetido à aprovação por Comitê de Ética em Pesquisa em Seres Humanos, Plataforma Brasil sob o número CAAE: 53362116.3.0000.5602 e atende a Resolução 466/12 CNS - Conselho Nacional de Saúde. Aprova as diretrizes e normas regulamentadoras de pesquisa envolvendo seres humanos.

\section{Resultados e discussão}

Para o estudo dos fatores associados à transmissão, levando em consideração os resultados sorológicos e moleculares, como também as informações referentes às características socioeconômicas, demográficas, individuais e comportamentais contidos nos questionários, foram traçados um diagnóstico dos casos de Hepatite $\mathrm{C}$, bem como os fatores de riscos associados ao qual está população se encontra exposta.

Com base em fundamentos estatísticos comprobatórios, foram feitas interpretações de significância e não significância a estes dados, que resultaram em dados sólidos, como também, os fatores de risco mais significativos para estas infecções virais no estado do Piauí.

A Tabela 1 apresenta dados de cruzamento da prevalência da infecção HCV, com variáveis sócio-demográficas obtidas dos questionários aplicados aos indivíduos portadores de HIV. Associação significativa foi observada apenas para a idade acima de 40 anos $(P=0,06)$, para o sexo masculino $(P=0,381)$, cor $(P=0,408)$, escolaridade $(P=0,276)$ e renda familiar $(P=0,395)$. 
Tabela 1 - Prevalência de HCV e variáveis sócio-demográficas dos indivíduos portadores de HIV no Piauí.

\begin{tabular}{cccccccc}
\hline Variáveis & HCV & HCV & Total & OR & IC (95\%) & (a) $\chi^{2}$ & $($ a)P \\
& $\begin{array}{c}\text { Positivo } \\
\text { Negativo }\end{array}$ & n & & & & \\
\hline
\end{tabular}

\section{Idade}

Mais de 40 anos

06

28

34

18 a 39 anos

05

74

79

$0,315 \quad 0,089-1,116$

3,46

0,06

Sexo

Masculino

08

85

93

Feminino

03

17

20

0,533

$0,128-2,218$

0,767

0,381

Cor

Branca

03

37

40

Preta/parda

08

65

73

0,659

$0,165-2,64$

0,352

0,408

\section{Escolaridade}

Até $1^{\circ}$ grau

09

69

78

A partir $2^{\circ}$ grau

02

33

35

2,152

0,440-10,526

0,933

0,276

\section{SituaçãoConjugal}

Com companheiro 06

32

38

Sem companheiro

05

70

75

2,625

0,746-9,239

2,389

0,115

\section{Renda Familiar em}

\section{salários mínimos}

Até 2 salários mín. 08

82

90

Ac. de 3 salários mín. 03

20

23

0,650

$0,158-2,675$

0,360

0,395

a) $\chi^{2}$ ( $P$ valor) e Teste Exato de Fisher quando necessário.

- Ac. - Acima

- Mín. - Mínimo

Fonte: LABMIC

As variáveis analisadas foram: sociodemográficas (idade, sexo, cor, escolaridade, situação conjugal e renda).

Carvalho et al. (2006), relataram que vários trabalhos do Brasil e de outros lugares do mundo mostram que a prevalência da coinfecção HCV/HIV varia e que o fator de risco principal para a aquisição do HCV é o uso de drogas injetáveis ilícitas, sendo a região Norte a de maior endemicidade. A ocorrência no estado do Piauí pode ser atribuída ao processo de miscigenação, em virtude da aproximação geográfica e o fluxo migratório entre os estados do Piauí e os estados da região Norte. 
A importância de se estudar os fatores de riscos envolvidos na coinfecção HIV/HCV é porque a progressão da Hepatite C é, muitas vezes, mais acelerada quando associada à infecção pelo HIV. É muito comum à associação da infecção pelo vírus da imunodeficiência humana (HIV) com a Hepatite C, principalmente pelo fato de as mesmas apresentarem os mesmos mecanismos de transmissão.

Calegari et al. (2011), relataram que em países em desenvolvimento, o uso de drogas injetáveis é um comportamento menos comum e a transmissão heterossexual é responsável pela maioria dos novos casos de HIV. Apesar de a transmissão sexual do HCV ainda não ser bem compreendida, a estimativa de prevalência da coinfecção HIV/HCV nos países em desenvolvimento baseia-se no principal fator de risco para a transmissão do HIV.

Segundo Silva \& Barone (2006), em relação às características sócio-demográficas dos pacientes, ser do sexo feminino é um fator de risco independente associado à infecção pelo HIV em pacientes infectados pelo HCV. Os principais mecanismos de transmissão do HIV em mulheres são a relação heterossexual e o uso de drogas injetáveis. Porém, no presente estudo o uso de drogas ilícitas e o hábito de compartilhar canudos e seringas são características relacionadas aos pacientes do sexo masculino, discordando da literatura citada. Assim, supõe-se que o risco de infecção pelo HIV nas mulheres esteja relacionado ao comportamento de seus parceiros sexuais.

No presente estudo a prevalência de HCV e variáveis sócio-demográficas dos indivíduos portadores de HIV no Piauí apresentou-se mais alta em indivíduos do sexo masculino (08), resultado que se encontra de acordo com os obtidos por Abreu et. al. (2013); Passadouro (2004); Marchesine et. al. (2007); Tovo et al. (2007), porém, Tovo et al. (2007) discorda apenas em relação à cor da pele (branca).

Amaral et al. (2007); Miranda et. al. (2004); Paraná et. al. (2007), descreveram que na população estudada, o sexo masculino foi predominante, faixa etária acima de 40 anos mostrando-se de acordo com os resultados obtidos nesta pesquisa. Já em relação à situação conjugal eram solteiros, discordando do presente estudo. Supõe-se que esses achados devem estar relacionados ao fato de a população masculina, principalmente solteiros, se expor mais a fatores de risco, como o sexo inseguro com múltiplos parceiros.

Em relação à cor da pele, este estudo encontrou o maior número de casos da coinfecção HCV em indivíduos pretos/pardos (08), discordando de Silva \& Barone, (2006), São Paulo, encontraram uma frequência maior em indivíduos brancos, procedente do estado de São Paulo e houve diferença estatística significativa em relação a renda mensal entre os grupos.

Quanto ao grau de escolaridade temos predominância de indivíduos que estudaram até o $1^{\circ}$ grau (09), resultado que se encontra de acordo com os obtidos por Silva e Barone, (2006); Araújo et al. (2005). Em relação à variável situação conjugal não foi encontrado resultado estatisticamente significante.

Em relação à renda e doenças sexualmente transmissíveis, o presente estudo se encontra coerente com a literatura Andrade et al. (2016); Amaral et al. (2013), os mesmos discordam com os resultados do presente estudo, em relação à renda familiar que se encontra inferior a dois salários mínimos.

Amaral et al. (2007), nota que muitos pacientes apresentam mais de um fator de risco associados para infecção pelos vírus HIV e HCV, sendo, portanto, difícil ter certeza da via pela qual adquiriram os vírus. Este fato se deve ao estilo de vida relacionado ao uso de drogas ilícitas injetáveis com compartilhamento de seringas, bem como a promiscuidade sexual, o intercurso sexual sem o uso de preservativos e a baixas condições sócio-econômicas.

Na Tabela 2 temos o cruzamento do HCV com as variáveis comportamentais, associadas a uma maior exposição à infecção pelo vírus HIV, sendo encontrada associação significativa apenas nas seguintes variáveis: ter realizado relacionamento sexual com usuário de ilícita $(P=0,048)$, cirurgia $(P=0,037)$, preferência sexual $(P=0,322)$ e DST $(P=0,407)$. 
Tabela 2 - Prevalência de HCV e variáveis associadas à maior exposição à infecção pelo vírus nos indivíduos portadores do HIV no Piauí.

\begin{tabular}{|c|c|c|c|c|c|c|c|c|}
\hline \multirow{2}{*}{$\begin{array}{l}\text { Variáveis } \\
\text { (a) } P\end{array}$} & Comportamento & $\mathrm{HCV}$ & $\mathrm{HCV}$ & Total & OR & IC $(95 \%)$ & (a) $\chi^{2}$ & \\
\hline & Evento & $\begin{array}{c}\text { Positivo } \\
\text { n }\end{array}$ & $\begin{array}{c}\text { Negativo } \\
\mathbf{n}\end{array}$ & $\mathbf{n}$ & & & & \\
\hline \multirow[t]{2}{*}{ Preferência Sexual } & (Hetero) & 09 & 71 & 80 & & & & \\
\hline & (Homo/Biss) & 02 & 31 & 33 & 1,965 & $0,401-9,627$ & 0,716 & 0,322 \\
\hline \multirow[t]{2}{*}{ Uso de preservativo } & Sim & 05 & 57 & 62 & & & & \\
\hline & Não & 06 & 45 & 51 & 0,658 & $0,189-2,295$ & 0,436 & 0,364 \\
\hline Contato sexual & Sim & 05 & 53 & 58 & & & & \\
\hline com prostitutas & Não & 06 & 49 & 55 & 0,770 & $0,221-2,686$ & 0,168 & 0,462 \\
\hline \multirow[t]{2}{*}{ Preso/Internado } & Sim & 02 & 40 & 42 & & & & \\
\hline & Não & 09 & 62 & 71 & 0,344 & $0,071-1,677$ & 1,881 & 0,148 \\
\hline Relac. Sexual com & Sim & 09 & 52 & 61 & & & & \\
\hline \multicolumn{9}{|l|}{ usuário de drogas } \\
\hline ilícita & Não & 02 & 50 & 52 & 4,327 & $0,891-21,019$ & 3,801 & 0,048 \\
\hline \multirow[t]{2}{*}{ Cirurgia } & Sim & 06 & 24 & 30 & & & & \\
\hline & Não & 05 & 78 & 83 & 3,900 & $1,093-13,914$ & 4,898 & 0,037 \\
\hline \multirow[t]{2}{*}{ Transfusão } & Sim & 03 & 22 & 25 & & & & \\
\hline & Não & 08 & 79 & 87 & 1,347 & $0,329-5,507$ & 0,172 & 0,463 \\
\hline \multirow[t]{2}{*}{ Tatuagem } & Sim & 04 & 33 & 37 & & & & \\
\hline & Não & 07 & 69 & 76 & 1,995 & $0,327-4,369$ & 0,073 & 0,514 \\
\hline \multirow[t]{2}{*}{ DST } & Sim & 07 & 51 & 58 & & & & \\
\hline & Não & 04 & 50 & 54 & 1,716 & $0,473-6,226$ & 0,686 & 0,407 \\
\hline
\end{tabular}

a) $\chi^{2}(P$ valor $)$ e Teste Exato de Fisher.

- Hetero - Heterossexual

- Homo/Biss - Homossexual/Bissexual

- Relac. - Relacionamento

Fonte: LABMIC 
As variáveis analisadas foram: comportamento sexual (heterossexual, homossexual e bissexual); situação dos parceiros sexuais (recebeu transfusão de sangue, uso de preservativo, preso/internado, relacionamento sexual com usuários de ilícita, cirurgia, tatuagem e DST).

Segundo Coelho et al. (2009), as variáveis comportamento homossexual/ bissexual e o contato sexual com mais de dois parceiros foram observados nos pacientes HIV-Aids. Contudo, após a análise e a regressão logística não se observou significância estatística em relação à coinfecção pelo HCV. Portanto, este trabalho revela a prevalência da coinfecção apontando uma maior significância entre as variáveis comportamento heterossexual e relacionamento sexual com usuários de ilícita, ter sido identificado em níveis próximos aos da significância $(P=0,048)$.

A população infectada com o HCV/HIV geralmente apresenta comportamento de alto risco para a transmissão de DSTs, pois, em geral, são pessoas que não praticam sexo seguro. Neste estudo esta variável não apresentou significância estatística, discordando dos dados encontrados por Araújo et al. (2005), que observaram alta frequência de não-uso ou uso irregular de preservativo entre adolescentes. Esses achados devem estar relacionados à falta de informação desses pacientes sobre transmissão de doenças por via sexual.

Martins (2011); Mendes-Corrêa et al. (2001), verificaram que a maioria dos pacientes eram heterossexuais. Esses achados, provavelmente, estão relacionados à maior prevenção de DSTs entre os homossexuais. Enquanto que a promiscuidade entre parceiros e a falta de prevenção de DSTs podem explicar o crescimento da taxa de infecção entre os heterossexuais. Porém, outros fatores de risco estão relacionados a hábitos sexuais que contribuem para maior transmissão do HCV, entre eles: maior número de parceiros sexuais, presença de DSTs, baixa adesão ao uso de preservativos. Além disso, a transmissão homem-mulher, concordando com os dados obtidos na presente pesquisa.

Este estudo não encontrou associação estatisticamente significativa entre as variáveis: situação conjugal com ou sem companheiro, uso de preservativo durante as relações sexuais, contato sexual com prostitutas, está preso/internado, ter realizado transfusão e tatuagem. Com relação à transfusão, a relação inversa ou negativa pela análise multivariada está longe de significar que este seja um fator protetor, pois não há plausibilidade biológica para este fato.

Os resultados de prevalência da coinfecção HIV/HCV no Estado do Piauí revelam uma baixa prevalência, quando comparados a outros centros brasileiros. Contudo, as variáveis: idade, sexo, cor, escolaridade, renda, preferência sexual, relacionamento sexual com usuário de ilícita, DST e cirurgia são os principais fatores de risco para a exposição ao HCV. Assim, levando-se em consideração estes dados, recomenda-se a implantação de políticas públicas que envolvam diagnóstico de rotina e aconselhamento aos pacientes portadores do HIV no Estado do Piauí, para melhor subsidiar as decisões clínicas, principalmente no que diz respeito à coinfecção HIV/HCV.

\section{Considerações Finais}

A partir dos dados obtidos nesse projeto, reforçam a necessidade de estratégias de saúde pública que se apoiem na intersetoridade, sobretudo para informar a Secretaria de Saúde do Estado do Piauí, a respeito dos casos de coinfecção HIV-Aids e HCV em usuários de drogas, que certamente irá proporcionar políticas de saúde voltadas especialmente para esta população no Estado do Piaú. Levando em consideração as questões sociais, econômicas e culturais deste grupo. Sobretudo entre a educação e assistência social, visando à educação em saúde e a oportunidade de uma assistência integral a esta população.

Conclui-se com o presente estudo que o sexo masculino é o fator de risco, independentemente associado com a infecção pelo HIV em pacientes infectados pelo HCV. Da mesma forma, que a cor preta/parda, relacionamento sexual com usuário de ilícita, preferência sexual, o uso pregresso ou atual de drogas ilícitas e o hábito de compartilhar seringas e canudos também se apresentaram como fatores de risco para a coinfecção HIV/HCV. Como perspectiva de trabalhos futuros, vislumbramos um trabalho sorológico georreferenciado no Estado do Piauí. 


\section{Referências}

Abreu, A. C. C., Simpaúba, B. G., Araújo, C. M. D. \& Araújo, T. M. E. (2013). Perfil clínico-epidemiológico dos casos de hepatite B e C do Piauí. R. Interd. Teresina, 6(4), 102-111.

Amaral, I. do S. A., Almeida, M. L. de, Alves, F. T., Móia, L. de J. M. P. \& Conde, S. R. S. S. (2007). Epidemiologia de pacientes co-infectados HIV/HCV atendidos na Fundação Santa Casa de Misericórdia do Pará. Revista Paraense de Medicina, 21(1), 15-20. http://scielo.iec.gov.br/scielo.php?script=sci_arttext\&pid=S0101-59072007000100003\&lng=pt\&tlng=pt.

Amaral, T. L. M., Rodrigues, A. U. \& Queiroz, M. M. D. C. (2013). Perfil clínico e epidemiológico da Hepatite C em Rio Branco, Acre, Brasil. Rev. Saúde. Com, 9(2), 64-79.

Andrade, A.P., Pacheco, S.D.B., Silva, F.Q. et al. (2017). Characterization of hepatitis B virus infection in illicit drug users in the Marajó Archipelago, northern Brazil. Arch Virol 162, 227-233. https://doi.org/10.1007/s00705-016-3060-z

Aquino, J. A., Pegado, K. A., Barros, L. P. \& Machado, L. F. A. (2008). Soroprevalência de infecções por vírus da hepatite B e vírus da hepatite C em indivíduos do Estado do Pará. Revista da Sociedade Brasileira de Medicina Tropical, 41(4), 334-337. https://doi.org/10.1590/S0037-86822008000400003.

Araújo, L. C. de., Fernandes, R. C. de S. C., Coelho, M. C. P. \& Medina-Acosta, E. (2005). Prevalência da infecção pelo HIV na demanda atendida no Centro de Testagem e Aconselhamento da Cidade de Campos dos Goytacazes, Estado do Rio de Janeiro, Brasil, 2001-2002. Epidemiologia e Serviços de Saúde, 14(2), 85-90. https://dx.doi.org/10.5123/S1679-49742005000200003.

Calegari, C. B., Oenning, R. T., Spillere, A. C., Trento, M. J. B., \& Fuzina, D. G. (2011). Perfil epidemiológico dos pacientes portadores do vírus da imunodeficiência humana (HIV) coinfectados com o vírus da hepatite C (HCV) no ambulatório de DST/AIDS da cidade de Criciúma. DST - J bras Doenças Sex Transm, 23(2): 90-94. DOI: 10.5533/2177-8264-201123207

Carvalho, F. H. P. de., Coêlho, M. R. C. D., Vilella, T. de A. S., Silva, J. L. A. \& Melo, H. R. de L. (2009). Co-infecção por HIV/HCV em hospital universitário de Recife, Brasil. Revista de Saúde Pública, 43(1), 133-139. https://doi.org/10.1590/S0034-89102009000100017.

Carvalho, F. H. P. de., Silva, A. N. M. R. da., Melo, H. R. de L. \& Coêlho, M. R. C. D. (2006). Prevalência do anti-HCV em pacientes soropositivos para o HIV. Revista Paraense de Medicina, 20(3), 11-13. http://scielo.iec.gov.br/scielo.php?script=sci_arttext\&pid=S0101-59072006000300003\&lng=pt\&tlng=pt..

Kuo, A. \& Gish, R. 2012. Chronichepatitis B infection. Clinics in liver disease, 16(2), 347-369. https://doi.org/10.1016/j.cld.2012.03.003

Martins, T., Narciso-Schiavon, J. L. \& Schiavon, L. de L. (2011). Epidemiologia da infecção pelo vírus da hepatite C. Revista da Associação Médica Brasileira, 57(1), 107-112. https://doi.org/10.1590/S0104-42302011000100024

Mendes-Corrêa, M. C. J., Barone, A. A. \& Guastini, C. (2001). Hepatitis C virus seroprevalence and risk factors among patients with HIV infection . Revista do Instituto de Medicina Tropical de São Paulo, 43(1), 15-19, 2001. https://www.revistas.usp.br/rimtsp/article/view/30475.

Miranda, E. C. B. M., Moia, L. de J. P., Amaral, I. do S. A., Barbosa, M. S. de B., Conde, S. R. S. da S., Araújo, M. T. F de., Cruz, E. do R. M. da., Demachki, S., Bensabath, G. \& Soares, M. do C. P. (2004). Infecções pelos vírus das hepatites B e C e o carcinoma hepatocelular na Amazônia oriental. Revista da Sociedade Brasileira de Medicina Tropical, 37(Suppl. 2), 47-51. https://doi.org/10.1590/S0037-86822004000700007

Nelson, P. K., Mathers, B. M., Cowie, B., Hagan, H., Des Jarlais, D., Horyniak, D., Degenhardt, L. (2011). Global epidemiology of hepatitis B and hepatitis $\mathrm{C}$ in people who inject drugs: results of systematic reviews. The Lancet. 13,378(9791):571-83. 10.1016/S0140-6736(11)61097-0.

Passadouro, R. (2004). Prevalência e factores de risco das Infecções por VIH, Hepatite B e C Num estabelecimento prisional de Leiria. Acta Méd. Port, 17: 381-384.

Pereira, A. S., et al. (2018). Metodologia da pesquisa científica. Ed. UAB/NTE/UFSM. https://repositorio.ufsm.br/bitstream/handle/1/15824/Lic_C omputacao_Metodologia-Pesquisa-Cientifica.pdf?sequence=1.

Reis, N. B. (2010). Conhecimento sobre HIV/AIDS entre usuários de drogas. 2010. 111p. DISSERTAÇÃO (Mestrado em Ciências, na área de Epidemiologia em Saúde Pública) Escola Nacional de Saúde Pública Sergio Arouca - ENSP.

Rodrigues, P. A., Dias, I., Lopes, A. M., Neves, R. P., Almeida, A., Abreu, R., Caria, E. (2007). Infecção por VIH e VHC em tóxico dependentes observados na consulta doenças infecto-contagiosas dum hospital geral de Lisboa. Revista Tóxico dependências, Edição IGT. Lisboa, 13(2), 63-68. http://www.sicad.pt/BK/RevistaToxicodependencias/Lists/SICAD_Artigos/Attachments/39/2007_02_TXT6.pdf

Santos, E. O., Coêlho, M. R. C. D., Vilella, T. A. S., Silva, J. L. A. \& Neto, E. P. A. L. (2008). Ocorrência e fatores de risco para co-infecção pelo vírus da hepatite $\mathrm{C}(\mathrm{HCV})$ em pacientes com o vírus da imunodeficiência humana (HIV) em Maceió, Brasil, Rev. para. med, 22(3)

Silva, A. C. M. \& Barone, A. A. (2006). Fatores de risco para infecção pelo HIV em pacientes com o vírus da hepatite C. Revista de Saúde Pública, 40(3), 482488. https://doi.org/10.1590/S0034-89102006000300017

Strazza, L., Massad, E., Azevedo, R. S. \& Carvalho, H. B. (2007). Estudo de comportamento associado à infecção pelo HIV e HCV em detentas de um presídio de São Paulo, Brasil. Cadernos de Saúde Pública, 23(1), 197-205. https://doi.org/10.1590/S0102-311X2007000100021

Tovo, C. V., Santos, D. E. dos., Mattos, A. Z. de, Mattos, A. A. de., Santos, B. R. \& Galperim, B. (2007). Avaliação da imunidade celular nos pacientes CoInfectados pelo vírus da hepatite C e vírus da imunodeficiência humana. Arquivos de Gastroenterologia,44(2), 113-117. https://doi.org/10.1590/S000428032007000200005 